\title{
Triggering on light-by-light scattering in the ATLAS experiment
}

\section{A. Ogrodnik*, on behalf of the ATLAS Collaboration}

AGH University of Science and Technology

E-mail: agnieszka.smaga@cern.ch

Light-by-light (LbyL) scattering is a quantum-mechanical process with a small cross-section, but possible to observe in ultra-peripheral high energy heavy-ion collisions. The signature of LbyL scattering consists of two low- $E_{\mathrm{T}}$ photons with absence of any other activity in the detector. Triggering on such events in the ATLAS experiment is challenging as the rate of LbyL events is tiny compared to hadronic inelastic interactions. Based on $2015 \mathrm{~Pb}+\mathrm{Pb}$ data, an evidence of $\mathrm{LbyL}$ scattering was reported by ATLAS in 2017. The starting point for developing the LbyL trigger for the upcoming $2018 \mathrm{~Pb}+\mathrm{Pb}$ run is a trigger strategy from the $2015 \mathrm{~Pb}+\mathrm{Pb}$ data taking. The work presented here is focused on lowering down the photon transverse energy requirement, which can provide almost a factor of two gain in the observed signal rate. In addition, trigger requirements need to be adjusted to the potentially larger instantaneous luminosity delivered by the LHC. This document presents an approach to address the challenges of triggering on LbyL scattering in the $2018 \mathrm{~Pb}+\mathrm{Pb}$ data taking.

Sixth Annual Conference on Large Hadron Collider Physics (LHCP2018)

4-9 June 2018

Bologna, Italy

${ }^{*}$ Speaker. 


\section{Introduction}

In addition to the main physics program based on proton-proton collisions, the ATLAS experiment [1] at the LHC also collects heavy-ion (HI) data with lead and xenon nuclei. Relativistic ions are a source of large electromagnetic fields. Therefore ultra-peripheral collisions provide a highly interesting class of events as they give an opportunity to study very rare processes involving two-photon interactions. An example of such a process is the light-by-light (LbyL) scattering a phenomenon which was theoretically postulated more than 80 years ago but due to its tiny cross section it had not been measured directly until 2017. Using lead-lead $(\mathrm{Pb}+\mathrm{Pb})$ collision data from 2015, the first evidence of this process was reported by the ATLAS Collaboration [2].

The LbyL scattering, $\gamma \gamma \rightarrow \gamma \gamma$, is a purely quantum-mechanical process. In the Standard Model, the virtual particles that mediate the LbyL coupling are electrically charged fermions or $W^{ \pm}$ bosons. In QED, the $\gamma \gamma \rightarrow \gamma \gamma$ reaction proceeds at lowest order in the fine structure constant $\left(\alpha_{\mathrm{em}}\right)$ via virtual one-loop box diagrams involving fermions, which is an $O\left(\alpha_{\mathrm{em}}^{4} \approx 3 \times 10^{-9}\right)$ process.

The LbyL final state is characterised by exactly two photons in back-to-back configuration (acoplanarity $=1-\Delta \phi_{\gamma \gamma} / \pi<0.01$, where $\Delta \phi_{\gamma \gamma}{ }^{1}$ denotes the difference in azimuthal angle between photons) with transverse energy, $E_{\mathrm{T}}$, and diphoton mass, $m_{\gamma \gamma}$, of the order of a few GeV. No other detector activity is expected in such events.

\section{Trigger strategy}

Despite the simplicity of the final state, triggering on LbyL is challenging due to the very low photon $E_{\mathrm{T}}$ requirement. In this kinematic region, apart from high rate of inelastic interactions, also electronic noise rate may be of the order of $\mathrm{kHz}$. To provide high efficiency, the designed trigger has to be inclusive. The trigger used in 2015 to collect data for the LbyL analysis required total transverse energy $\sum E_{\mathrm{T}}^{\mathrm{L} 1}$, between 5 and $200 \mathrm{GeV}$ at the hardware-based Level-1 trigger and no more than 1 hit in the inner ring of Minimum Bias Trigger Scintillators and maximum of 10 hits in the Pixel detector at the software-based high-level trigger (HLT). This set of trigger requirements enabled the measurement of LbyL for final-state photons with $E_{\mathrm{T}}>3 \mathrm{GeV}$. As LbyL scattering cross-section decreases with photon $E_{\mathrm{T}}$, the aim is to improve the trigger strategy to be able to lower the threshold from $3 \mathrm{GeV}$ to $2.5 \mathrm{GeV}$. To improve detector sensitivity to low- $E_{\mathrm{T}}$ particles the Level1 calorimeter noise settings have been optimised. The impact of these improved settings on the Level-1 trigger performance is presented here along with the optimisation of $\sum E_{\mathrm{T}}^{\mathrm{L} 1}$ requirements.

\section{Event characteristics and selection}

Performance studies of low- $E_{\mathrm{T}}$ diphoton triggers can be conducted using exclusive pairs of electrons. Its high production cross-section provides enough statistics for an efficiency measurement. At Level-1 trigger, which does not include tracking information, such events have a similar

\footnotetext{
${ }^{1}$ ATLAS uses a right-handed coordinate system with its origin at the nominal interaction point (IP) in the centre of the detector and the $z$-axis along the beam pipe. The $x$-axis points from the IP to the centre of the LHC ring, and the $y$-axis points upward. Cylindrical coordinates $(r, \phi)$ are used in the transverse plane, $\phi$ being the azimuthal angle around the $z$-axis. The pseudorapidity is defined in terms of the polar angle $\theta$ as $\eta=-\ln \tan (\theta / 2)$.
} 
detector signature as LbyL photons. The similarities between final states of these two processes include low $E_{\mathrm{T}}$ of products, good correlation between particles $E_{\mathrm{T}}$, acoplanarity $<0.01$ and minor detector activity. Evaluation of the trigger efficiency for the improved calorimeter noise settings at Level-1 is performed with the test data sample from xenon-xenon $(\mathrm{Xe}+\mathrm{Xe})$ collisions at $\sqrt{s_{\mathrm{NN}}}=5.44 \mathrm{TeV}$, collected in October 2017. Exclusive $e^{+} e^{-}$events for an efficiency measurement are extracted from a minimum-bias sample selected with the logical OR of two triggers, first one requiring $\sum E_{\mathrm{T}}^{\mathrm{L} 1} \leq 4 \mathrm{GeV}$ at Level-1 and at least one track at HLT and a second one requiring $\sum E_{\mathrm{T}}^{\mathrm{L} 1}>4 \mathrm{GeV}$. The selection criteria include exactly two oppositely-charged offline tracks with transverse momentum, $p_{\mathrm{T}}>1 \mathrm{GeV}$, in back-to-back configuration (acoplanarity $\left.<0.2\right)$. These tracks must be matched to an offline electromagnetic (EM) clusters with $\Delta R=\sqrt{(\Delta \eta)^{2}+(\Delta \phi)^{2}}<$ 0.7 , where $\Delta \eta$ and $\Delta \phi$ denote the difference in pseudorapidity and azimuthal angle, respectively, between the track and the EM cluster. Minor detector activity, described by the sum of $p_{\mathrm{T}}$ of remaining tracks, is required to be less than $2 \mathrm{GeV}$. After applying these criteria, 72 event candidates are selected. Left panel of Figure 1 shows the acoplanarity distribution of the selected exclusive $e^{+} e^{-}$candidates while right panel demonstrates the good correlation between the $E_{\mathrm{T}}$ of the EM clusters associated to electron candidates, $E_{\mathrm{T}}^{\text {cluster1,2. }}$.
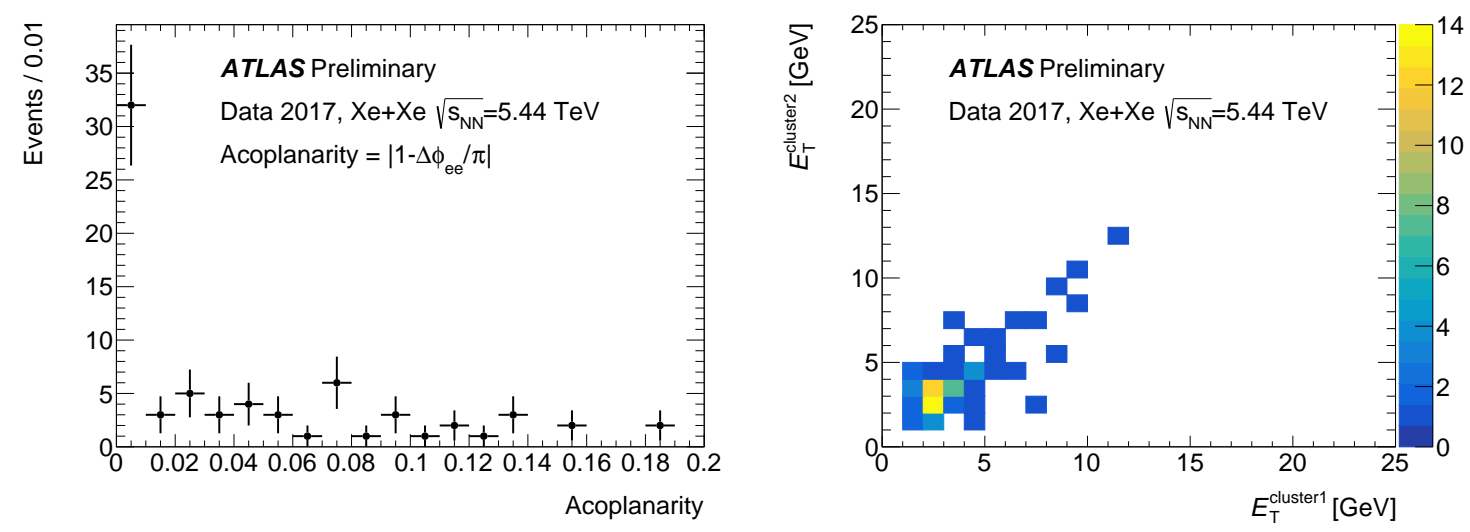

Figure 1: Acoplanarity distribution of two tracks (left panel) and correlation between transverse energy of two EM clusters matched to offline tracks for the exclusive $e^{+} e^{-}$event candidates in $\mathrm{Xe}+\mathrm{Xe}$ data [3].

\section{Trigger efficiency in $2015 \mathrm{~Pb}+\mathrm{Pb}$ and $2017 \mathrm{Xe}+\mathrm{Xe}$}

The trigger efficiency in $2015 \mathrm{~Pb}+\mathrm{Pb}$ data was measured using exclusive $e^{+} e^{-}$events passing an independent trigger [4]. It is usually presented as a function of the sum of $E_{\mathrm{T}}$ of the two EM clusters corresponding to the $e^{+} e^{-}$pair. The Level-1 trigger efficiency is measured for one dedicated item available in 2015: L1 TE5, which requires $\sum E_{\mathrm{T}}^{\mathrm{L} 1}>5 \mathrm{GeV}$. The efficiency reaches $20 \%$ for $E_{\mathrm{T}}^{\text {cluster1 }}+E_{\mathrm{T}}^{\text {cluster2 }}=5 \mathrm{GeV}$, and $\sim 70 \%$ for $E_{\mathrm{T}}^{\text {cluster1 }}+E_{\mathrm{T}}^{\text {cluster2 }}=6 \mathrm{GeV}$, and $100 \%$ for $E_{\mathrm{T}}^{\text {cluster1 }}+E_{\mathrm{T}}^{\text {cluster2 }}=8 \mathrm{GeV}$.

In the $2017 \mathrm{Xe}+\mathrm{Xe}$ data, trigger efficiency is measured for two Level-1 items: L1 TE4 and L1 TE5, requiring $\sum E_{\mathrm{T}}^{\mathrm{L} 1}>4$ or $5 \mathrm{GeV}$, respectively. It is presented as a function of the sum of $E_{\mathrm{T}}$ of the two EM clusters matched to the $e^{+} e^{-}$candidates. The L1 TE4 trigger is fully efficient starting 
from $5 \mathrm{GeV}$, while L1 TE5 starting from $8 \mathrm{GeV}$. As it is shown in Figure 2, both triggers turn on faster and L1 TE4 has a higher efficiency in comparison to the trigger used in $2015 \mathrm{~Pb}+\mathrm{Pb}$ data taking.
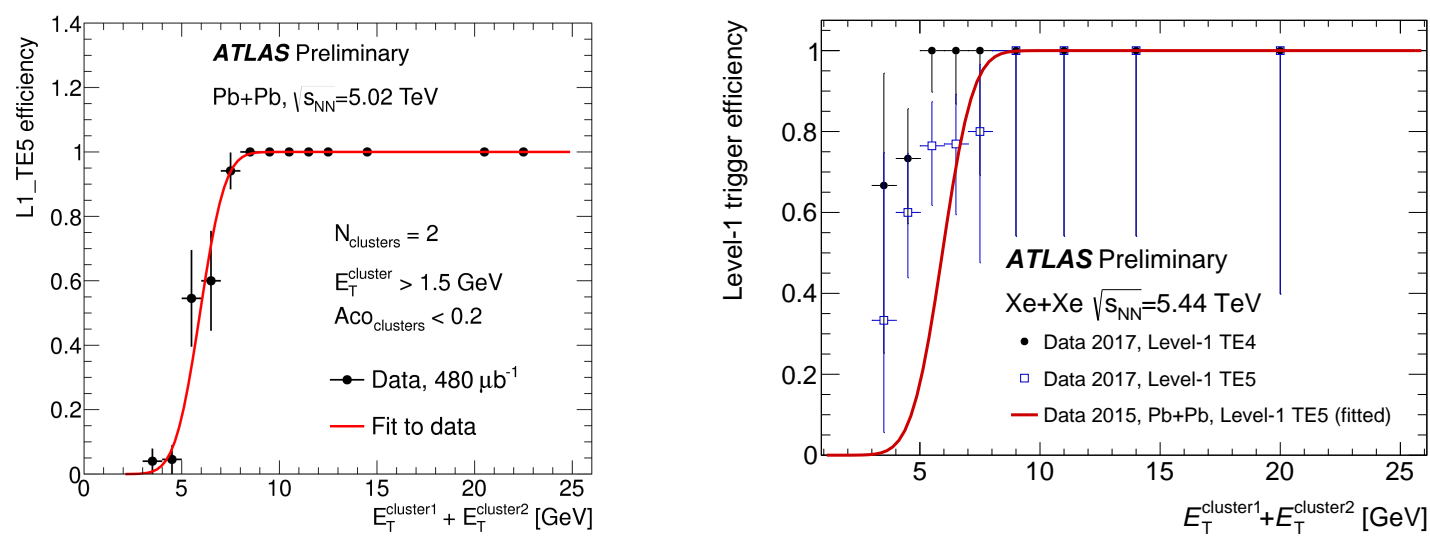

Figure 2: Efficiency of Level-1 triggers: L1 TE4 (full circles) and L1 TE5 (open squares) as a function of the sum of transverse energy of two EM clusters satisfying the exclusive $e^{+} e^{-}$event selection in $2017 \mathrm{Xe}+\mathrm{Xe}$ data [3] (right panel) and efficiency of L1 TE5 for exclusive $e^{+} e^{-}$events in $2015 \mathrm{~Pb}+\mathrm{Pb}$ data [4] (left panel). Triggers L1 TE4 and L1 TE5 have $4 \mathrm{GeV}$ and $5 \mathrm{GeV}$ thresholds on $\sum E_{\mathrm{T}}^{\mathrm{L} 1}$, respectively. The fit represents a $\mathrm{L} 1 \mathrm{TE} 5$ trigger efficiency measured in $2015 \mathrm{~Pb}+\mathrm{Pb}$ data.

\section{Trigger rates}

The optimization of the calorimeter noise settings in terms of the efficiency results in an increase of the rate of the total energy triggers. It is necessary to reduce the rate which can be achieved by vetoing events with large $\sum E_{\mathrm{T}}^{\mathrm{L} 1}$. In $2015 \mathrm{~Pb}+\mathrm{Pb}$, a cut $\sum E_{\mathrm{T}}^{\mathrm{L} 1}<200 \mathrm{GeV}$ decreased the rate of $\mathrm{L} 1 \mathrm{TE} 5$ by a factor of 3.5. In Figure 3 a comparison of $\sum E_{\mathrm{T}}^{\mathrm{L} 1}$ distributions between exclusive $e^{+} e^{-}$ events and minimum-bias sample is shown. In selected $e^{+} e^{-}$events, the largest value observed for $\sum E_{\mathrm{T}}^{\mathrm{L} 1}$ is $25 \mathrm{GeV}$. Because of the limited statistics of the test sample, the maximum $\sum E_{\mathrm{T}}^{\mathrm{L} 1}$ threshold should be set higher than this value, at about $100 \mathrm{GeV}$, to give also the possibility to establish limits for BSM searches [5].

\section{Acknowledgments}

This work was supported in part by Polish National Science Centre grant DEC-2016/23/B/ST2/01409, by the AGH UST grant no. 15.11.220.717/4 within subsidy of the Ministry of Science and Higher Education, and by PL-Grid Infrastructure. 


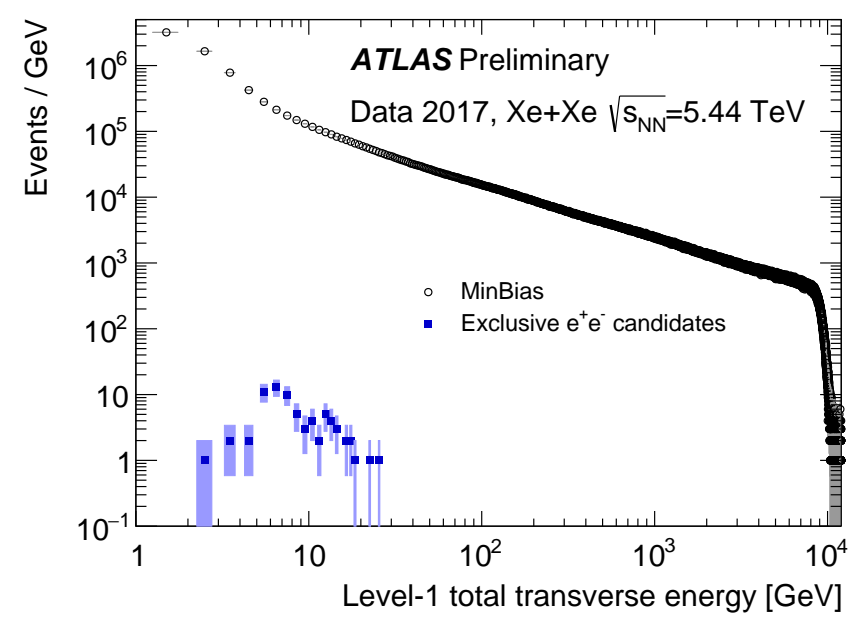

Figure 3: Distribution of $\sum E_{\mathrm{T}}^{\mathrm{L} 1}$ in minimum-bias events (black circles) and exclusive $e^{+} e^{-}$event candidates (blue squares) in $2017 \mathrm{Xe}+\mathrm{Xe}$ data [3].

\section{References}

[1] ATLAS Collaboration, The ATLAS Experiment at the CERN Large Hadron Collider, JINST 3 (2008) S08003

[2] ATLAS Collaboration, Evidence for light-by-light scattering in heavy-ion collisions with the ATLAS detector at the LHC, Nature Physics 13 (2017) 852 [hep-ex/1702.01625]

[3] https://twiki.cern.ch/twiki/bin/view/AtlasPublic/HeavyIonTriggerPublicResults

[4] ATLAS Collaboration, Light-by-light scattering in ultra-peripheral $P b+P b$ collisions at $\sqrt{s_{N N}}=5.02$ TeV with the ATLAS detector at the LHC, ATLAS-CONF-2016-111, http://cdsweb.cern.ch/record/2220773

[5] S. Knapen, T. Lin, H. K. Lou, and T. Melia, Searching for Axionlike Particles with Ultraperipheral Heavy-Ion Collisions, Physical Review Letters 118 (2017) 171801 [hep-ph/1607.06083] 